\title{
El Ms. 229 (PN7) de la 'Bibliothèque Nationale' de París; base de las ediciones modernas del Laberinto de Fortuna de Juan de Mena
}

\section{The Ms. 229 (PN7) of the 'Bibliothèque Nationale' of Paris; Basis of Juan de Mena's Laberinto de Fortuna Modern Editions}

\author{
MAXIM P.A.M. KeRKHOF \\ Universidad Católica, Nimega, Holanda
}

El artículo analiza y describe el manuscrito 229 (PN7) de la Bibliothèque Nationale de París, que ha sido la base de todas las ediciones modernas del Laberinto de Fortuna de Juan de Mena desde la de John Cummins en 1968. El análisis constituye una muy pormenorizada descripción de todos los ángulos ecdóticos necesarios para comprender y apreciar este valioso códice, desde tipos de letra, los posibles copistas, el soporte de pergamino, las filigranas, las capitulares, la disposición del texto central y textos menores que lo acompañan, así como la trayectoria textual del códice, entre otros aspectos.

Palabras Clave: ecdótica, crítica textual, manuscrito, códice, biblioteca nacional de París, Juan de Mena, Laberinto de Fortuna

The paper analyzes the manuscript 229 (PN7) of the National Library in Paris, which is the basis of all Juan de Mena's Laberinto de Fortuna modern editions, the first one of them was John Cummins' in 1968. The article offers a detailed description of this codex from many ecdotic angles, such as the paleographic type of letter, the possible copyists, the texts included in this codex, besides Juan de Mena's poem, the filigree works and watermarks, the capital letters heading some texts and parts, the textual trajectory or path followed by the codex, etc.

KEYWORDS: textual critic, ecdotic discipline, manuscript, codex, national library of Paris, Juan de Mena, Laberinto de Fortuna

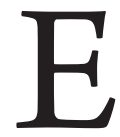

ste manuscrito es la base de todas las ediciones modernas a partir de la de John Cummins de $1968 .{ }^{1}$ En 1958 Florence Street ("The Text”, 63-71) subrayó la importancia de este códice describiéndolo

${ }^{1}$ Se trata de las ediciones de John Cummins (Salamanca, 1968; Madrid, 1979), Louise 
someramente. En más de una ocasión llamé la atención hacia algunos errores cometidos por la malograda hispanista inglesa.

Sin embargo, falta todavía un estudio detallado de este manuscrito parisiense. El códice (PN7) ${ }^{2}$ se conserva en la 'Bibliothèque Nationale' de París, con la signatura Esp. 229 (ant. 844/7823). ${ }^{3}$ Contiene 81 folios, escritos por varias manos. Sus dimensiones son 295 X $210 \mathrm{~mm}$. Fue encuadernado en el siglo XVIII en marroquí de color rojo, y lleva el blasón de Luis $\mathrm{XV}^{4}{ }^{4}$ Está rotulado "Labor. de fortu."

En la primera hoja está escrito "Volume de 81 feuillets/23 Septembre 1847 ", y en la segunda se lee "Espagnol/Labyrinte de fortune de Jean du mayne”. La tercera y cuarta hoja están en blanco. La foliación comienza en la quinta hoja. A la vuelta de este folio (1v) se halla un dibujo (exlibris) que representa la puesta del sol, con las palabras "LE IOVR/SEN VA".5 Por debajo está escrito "Des marieuz". Además del Laberinto (fols. 2r-76v), escrito en varios tipos de letra redonda de la segunda mitad del siglo Xv, figuran en el manuscrito tres poesías: la primera en catalán, y la segunda y tercera en castellano, y una glosa en prosa en catalán del comendador Stela (fols. $77 \mathrm{r}-81 \mathrm{v}$ ), escritas en letra bastarda, las cuales muy probablemente fueron añadidas más tarde. Las filigranas son un losange, con las barras de Aragón, sosteniendo una corona, común en Aragón a partir de 1427 (Bofarull y Sans, Heraldic Watermarks, $\left.\mathrm{n}^{\circ} 103\right),{ }^{6}$ y una mano con una estrella, común en la Provenza entre 1446 y 1473 (Briquet, Les filigranes, $n^{\circ}$ 11090; Aviñón, 1446).

En el Laberinto intervinieron seis manos, cuatro copistas, A, B, C y D, que usaron diferentes tipos de letra redonda, y dos comentaristas, $\mathrm{A} y \mathrm{~B}$, que escribieron en letra bastarda. ${ }^{7} \mathrm{El}$ esquema siguiente muestra la distribución de las diferentes manos:

Vasvari Fainberg (Madrid, 1976), Miguel Angel Pérez Priego (Madrid, 1976; Madrid, 1989; Barcelona, 1989) y de José María Azáceta (Barcelona, 1986).

${ }^{2}$ Sigo las siglas utilizadas por Dutton en su Catálogo-Índice.

${ }^{3}$ Véanse Ochoa, Catálogo razonado, $\mathrm{n}^{\circ} 7823$ y A. Morel-Fatio, Catalogue, n ${ }^{\circ} 589$ ).

${ }^{4}$ El códice PN5, el Ms. 227 de la 'Bibliothèque Nationale' de París lleva la misma encuadernación.

${ }^{5}$ Según Aubrun ("Inventaire des sources", 308) el dibujo se añadió en el siglo Xvi. El mismo dibujo (exlibris) se encuentra también en el manuscrito 228 (PN6) de la misma biblioteca.

${ }^{6}$ La misma filigrana se encuentra también en el ms. SA5, el 2244 de la Biblioteca Universitaria de Salamanca.

${ }^{7}$ En su estudio "The Text", 67-68, Florence Street sostuvo que la confección del texto del Laberinto fue la obra de 'dos' copistas y dos comentaristas; sin embargo, veo en sus papeles que más tarde rectificó esta opinión, porque en ellos habla también de 'cuatro' copistas.

Medievalia 50, 2018, pp. 217-231 
TEXTO

copista A:

copista B:

copista C:

copista D:

\section{GLOSAS}

c. 1-195 (fol. 49v) c. 8, 37, 38,51-53, $60,63,65,71,73,75,76,78,81,83$, $86,88,89,93,96,102,106,110,111$, $116,119,122,123,1$ 26-129, 139144, 147-151, 157, 159, 163, 164, 185.

c. 196-232(fol.59v)c.196,191,197,201, 203, 207, 209, 210, 215-218, 220-222.

c. $233-272$ (fol.69v) c. $165-172,194$

c. 273-300 (fol.76v).

Casi todas estas glosas figuran también en BC3, ML2, S V2, y una parte de ellas en MM1. ${ }^{8}$

${ }^{8}$ El Laberinto figura en los manuscritos siguientes: BC3Barcelona, Biblioteca de Catalunya, ms. 1967.

BM1Montserrat, Biblioteca de la Abadía, ms. 992.

BM2Montserrat, Biblioteca de la Abadía, ms. 1134.

CO1Coimbra, Biblioteca da Universidade, ms. 1011.

GB1Cologny (Suiza), Biblioteca Bodmeriana, Cod. Bodmer 45.

HH1 Harvard, Houghton Library, Ms. Sp. 97.

LB2Londres, British Library, Add. 33383.

MH1Madrid, Real Academia de la Historia, 2 MS 2.

ML2Madrid, Biblioteca de la Fundación Lázaro-Galdiano, ms. 208.

MM1Madrid, Biblioteca de Bartolomé March, ms. 20-5-6.

MN6bMadrid, Biblioteca Nacional, ms. 2882.

NH5Nueva York, Biblioteca de la 'Hispanic Society' ms, HC 397/703.

PN5París, Bibliothèque Nationale, Esp. 227.

PN7París, Bibliothèque Nationale, Esp. 229.

SA5Salamanca, Biblioteca Universitaria, ms. 2244.

SM1Santander, Biblioteca de Menéndez y Pelayo, ms. 70.

SV2Sevilla, Biblioteca Colombina, ms. 83.6.10.

El stemma que he elaborado de estos representantes manuscritos presenta el gráfico siguiente:

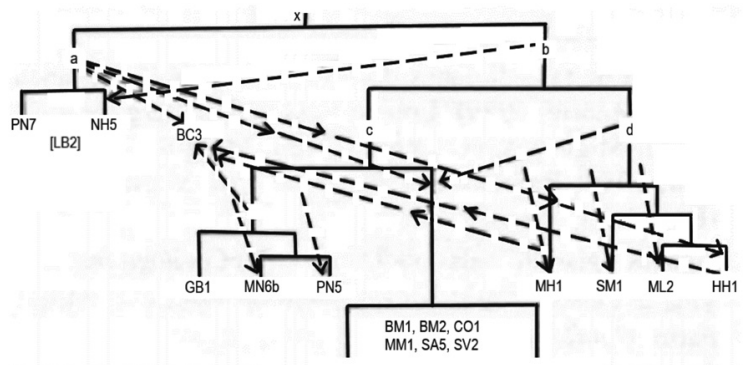


El comentarista A escribe las glosas a las coplas 2-8, 11, 13, 15, 18, 27, $28,31,34-43,45-49,51-54,60,62-66,72,75,77,79-80,82,84-94,102-104$, 110, 112. 115, 196, 215, 216, 241-243, 245-252, 275.

Los comentarios que B añade a las coplas 289, 295 y 296 son exactamente iguales a los de las ediciones de Hernán Núñez (Sevilla, 1499; Granada, 1505).

A veces el copista $A$ escribe el reclamo y el copista $C$ el texto de la glosa (por ej. en las glosas de las estr. 167 y 168). También ocurre que el comentarista A amplía las glosas escritas por los copistas A y B.

Este comentarista A repasó el texto entero añadiendo notas eruditas, corrigiendo palabras y cambiando a veces el orden de palabras, versos y estrofas con la indicación de las letras $b$ y $a$. En sus glosas se refiere muchas veces a la literatura clásica. Los autores más citados son Virgilio (Eneida, Geórgicas), Ovidio (Metamorfosis y Ars amandi), y Lucano (Farsalia); además hay referencias a Estrabón (De situ orbis), Pomponio Mela, Juvenal, César, Marcial y Horacio. El comentarista A fue el primero en señalar el influjo de las Metamorfosis, V, vss. 346 y sigs. con respecto a la descripción de Sicilia en la estr. $53,{ }^{9}$ y el de la historia de Ericto, la hechicera de Tesalia, de la Farsalia, VI, vss. 604 y sigs. para el episodio de la maga de Valladolid (estrofas 241 y sigs.). ${ }^{10}$

Florence Street (“The Text”, 68) opinó que los copistas y el comentarista $\mathrm{A}$ :

were working together from a MS. of the Laberinto consisting of both poem and commentary and originally supervised by Mena himself. We have no means of finding out how far Commentator A altered the commentary, but it is quite certain that he and the two scribes produced a text which is nearer to the original than any of the others that have come down to us.

Hace años defendí la tesis de que los copistas trabajaron sobre un mismo manuscrito y que más tarde un comentarista culto - el "Commentator A”- añadió sus anotaciones (Kerkhof, “Hacia una nueva edición”, 182-183). Los argumentos que la hispanista inglesa adujo en favor de su tesis son:

1. las manos tienen muchas características en común.

2. los sistemas ortográficos y de abreviación son muy parecidos.

3. a veces los copistas raspan un vocablo y lo reemplazan por otro que el comentarista A aprueba en su glosa.

\footnotetext{
${ }^{9}$ Cf. María Rosa Lida de Malkiel, Juan de Mena, 36.

${ }^{10} \mathrm{Cf}$. las ediciones del Laberinto de Núñez (Sevilla, 1499) y del Brocense (Salamanca, 1582).
} 
4. a menudo el comentarista A amplía una nota/glosa escrita por un copista, y hasta cambian las manos una vez en medio de una palabra, en la glosa a la copla 75: "de sus vidas y estos dos [dichos, según Street] ermanos eran quatro" (la parte en letra cursiva representa lo añadido por el comentarista).

No creo que estos argumentos sean contundentes, porque:

- lo observado en 1. y 2. sólo vale para los 'copistas'.

- con respecto al tercer argumento, es igualmente posible que el comentarista A raspase vocablos y los reemplazase por otros.

- en la glosa a la copla 75 se ve claramente que el copista A escribió:

Cosa de fama / gosa por

fama de sus fechos avnque

non por vista de sus vidas.

El comentarista A tacha das (última línea) y continúa la nota tras vi-: "das y estos [das tachado] ermanos eran quatro, el rrey don alfonso de aragón, etc." Véase la foto, más abajo. Resulta, pues, que también en este caso el comentarista amplía la nota escrita por el copista: ${ }^{11}$

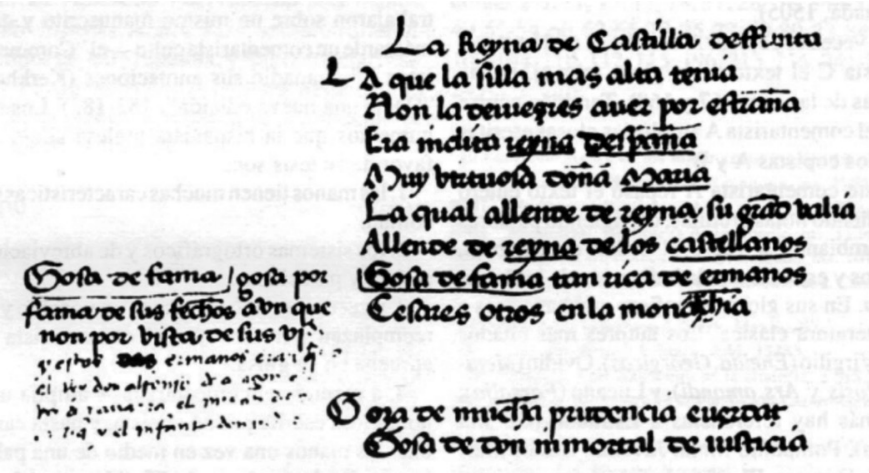

Street ("The Text", 68) se equivocó también al escribir que "both Scribe $\mathrm{A}$ and Commentator A refer repeatedly to information contained 'en otro

\footnotetext{
${ }^{11}$ Así, por ejemplo, añade a la glosa sobre Alcides (copla 65) otra explicación: "o se dize alcides de alceo su auuelo putativo padre de amphitrion".
}

Medievalia 50, 2018, pp. 217-231 
libro'”, puesto que las seis referencias al 'otro libro' son todas del comentarista A. ${ }^{12}$ Por lo tanto, sostengo que los copistas transcribieron su texto y glosas de un modelo y que el erudito comentarista añadió más tarde sus anotaciones, amplió a veces una glosa escrita por uno de los copistas, e introdujo algunas correcciones, teniendo a su disposición 'otro libro', otra copia del Laberinto, a que alude de vez en cuando en sus glosas.

Las glosas escritas por el comentarista A no son de Mena, porque por ejemplo en la glosa al interlineado 'austro' (vs. 85) se lee: "el austro ha de dezir vno d'estos [...]"; y en la glosa a la estrofa LXIV critica al poeta: "Manseolo: esta fue artemisa; su ystoria es vulgar e la descriue valerio más a propósito de las palabras aquí scriptas [...]”.

La presencia de numerosos rasgos catalano-aragoneses muestra que el ms. 222 PN7 fue copiado en Cataluña (cf. las marcas del papel), o tal vez en la corte aragonesa de Nápoles, de donde proceden varios de los cancioneros del fondo español de la 'Bibliothèque Nationale' de París. ${ }^{13}$ Después de la muerte de Ferrante I (en 1494), que en 1458 había sucedido a su padre Alfonso V en el trono de Nápoles, Carlos VIII llevó buena parte de los manuscritos y libros coleccionados por Alfonso $\mathrm{V}$ y Ferrante para la biblioteca de la corte napolitana a Francia, depositándolos en la biblioteca de Blois; más tarde fueron trasladados a Fontainebleau. ${ }^{14}$ Ahora bien, dondequiera que se copiase el manuscrito en cuestión, en todo caso parece muy probable que estuviera en Nápoles, donde un erudito italiano - el comentarista $\mathrm{A}$ - , que probablemente trabajó en la corte napolitana de Ferrante, lo repasó, corregiéndolo y anotándolo donde lo creía necesario. Buena prueba de ello son los muchos italianismos ${ }^{15}$ que figuran en las glosas de este anotador. En favor de esta hipótesis se pudiera aducir también la referencia que el comentarista A en su glosa al vs. 114 hace al Roma restaurata (por Roma instaurata, obra compuesta entre 1444 y 1446) del humanista italiano Flavio Biondo; ${ }^{16}$ la cita — en italiano- de unos versos de la Rima CXXXV de Petrarca en su glosa sobre la fuente de Epiro (estr. 45): "messer

${ }^{12}$ Cf. las coplas 6 [pierio subsidio], 11 [las naues], 63 [con vulto más pío], 79 [de los corneles], 243 [fasila; por ¿fabila?] y 275 [ojos de alas de dragos]. Dos veces (coplas 18 y 89 ) el comentarista apunta en el margen otras lecturas (respectivamente venido en extremo y trucidas), tomadas sin duda también del 'otro libro'. Por lo general, corresponden estas lecciones a las de la tradición $b$.

${ }^{13}$ Cf. Maxim. P.A.M. Kerkhof, en la introducción a su edición de la Comedieta de Ponza, 52-55; y Robert Black, "Poetic Taste", 167-174.

${ }^{14}$ Cf. Dorothy S. Severin y Fiona Maguire, “The Spanish Songbook Project”, 53.

${ }^{15}$ Por ejemplo fluvio, marciale, vulgare, preguntato, capellyo < it. capello, etc.

${ }^{16}$ Cf. también Julian Weiss, The Poet's Art, 127, nota 29. 
francisco petrarca en vna su cançion la conmemora en aquestas seguintes palabras vna fonte ha en epyro de cuy si lege che essendo freda ella omne spenta facella Accende et spenge qual trouasse accesa"; ${ }^{17}$ o lo que escribe en la glosa al vs. 701: "[...] el amiento quiere dezir en ytaliano la zagallya $[. .$.$] ”.$

El comentarista A sustituye de vez en cuando una lección de PN7 por otra superior, la que también está presente en la tradición $b$, dejándose guiar muy probablemente por el 'otro libro', otro manuscrito del Laberinto al que algunas veces alude en sus anotaciones, como ya he mencionado arriba. ${ }^{18}$ Por ejemplo en el verso 61 (estr. VII) todos los manuscritos, excepto BC3, BM1 y PN7, leen 'e las siete Pleyas que Atlas otea. Todavía se puede ver bien que PN7 leía originalmente 'e las siete Pleyas que quin [catalán por 'quien'] las otea'. Por ser ésta también la lección de BC3 podemos concluir que es la de la tradición $a .{ }^{19} \mathrm{El}$ comentarista cambia 'quin las' en atlas, tachando 'qui', corrigiendo la 'n' en 'a' y poniendo una ' $t$ ' entre la 'a' y la 'l' de 'las'; además continúa la glosa sobre las pleyas, escrita en letra del copista A: "y dize que atlas las otea y mira porque atlas fue gran astrólogo e por ende las dichas pleyas se dizen fijas de atlas y syempre se asconden la mañana venida la bruma y ansý cada vna de las sopra [italianismo] dichas guarda su ley":

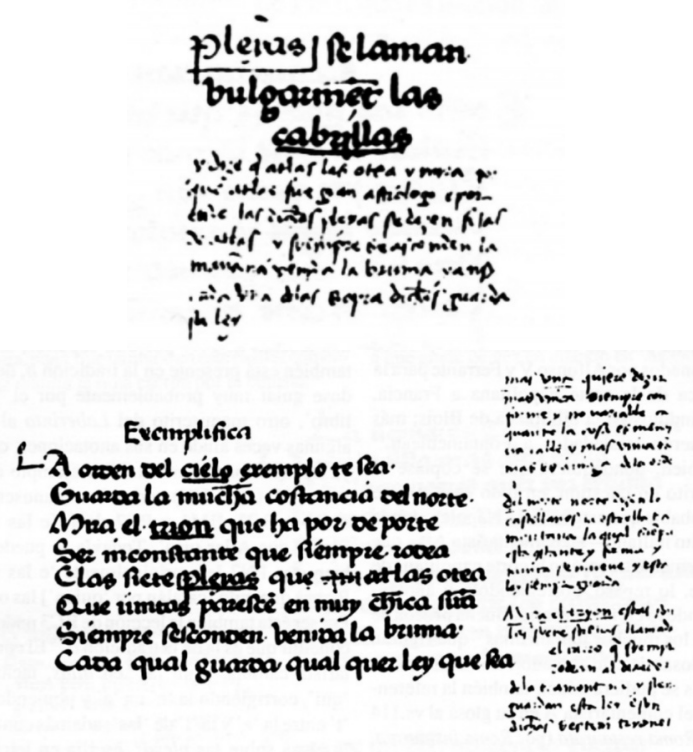

${ }^{17}$ Cf. Francesco Petrarca, Rima CXXXV: “Un' altra fonte ha Epiro / Di cui si scrive ch' essendo fredda ella, / Ogni spenta facella / Accende, e spegne qual trovasse accesa” (vss. 6164). Para las formas spenge (mod. spegne) y omne (mod. ogni, que probablemente deriva de omne $[m]$ ), véase Nicola Zingarelli, Vocabulario, s.v. 'spengere' y 'ogni'.

${ }^{18}$ En sus glosas a las estrofas 6, 11, 63, 79, 243 y 275.

${ }^{19}$ El copista de NH5 tomó la lección 'que adlas otea' de la otra tradición. 
En el verso 85 subsana mediante un interlineado la mala lectura norte en austro, siendo esta también la lección de CO1, GB1, MH1, MN6b y PN5, y en una glosa aclara: "el austro ha de dezir vno d'estos, el qual es uiento meridional contrario al bóreas, aquí norte llamato [fíjese en la forma italianizante]”:
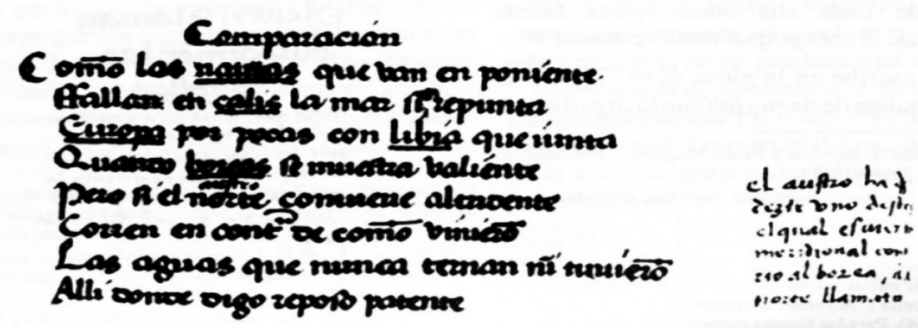

Partiendo de la edición de Hernán Núñez (Granada, 1505), el otro comentarista (B) también repasó el texto corrigiéndolo frecuentemente; en varias enmiendas se reconoce fácilmente su letra. ${ }^{20} \mathrm{Tal}$ vez introdujese también cambios mediante la modificación de letras, la añadidura de letras o correcciones sobre una raspadura. Está claro que en estos casos no se puede saber si una corrección es de uno de los copistas o del comentarista A o B. Pero lo que sí me parece perfectamente defendible es que las correcciones que van acompañadas de una glosa en letra del comentarista A se deben a su intervención, y que las correcciones sobre formas originales todavía visibles, siendo estas comunes a BC3 y NH5, a NH5, a BC3, o a toda la tradición manuscrita, son también de la mano de uno de los comentaristas. En estos casos muchas veces se trata de lecturas únicas de PN7, siendo las lecturas que la tradición manuscrita propugna igualmente aceptables o mejores; $y$, claro está, entonces no conviene seguir al PN7, lo que los editores modernos sí hacen en la mayoría de los casos. Un buen ejemplo se encuentra en la descripción de Sicilia en la estrofa LIII del Laberinto. En estos versos Mena amplía la información

\footnotetext{
${ }^{20}$ Basándose en Hernán Núñez, el comentarista B introdujo los siguientes interlineados: cobijando (vs.48), planco, apollodoro (vs.950), phrygineta (vs.968), pisuerga (vs.1289), por (vs.1298), luque (vs.2262), rescate (vs.2285), alcaudete (vs.2293), y cañete (vs.2305), bencayde (vs.2311), benemexi (vs.2312), fatidica (vs. 2332), quando serian (vs.2338), apacados (vs.2340), veras (vs.2362), tormentos (vs. 2384), plazen (vs.2399), mundo (vs.2400). Con toda probabilidad corrigió también muchas en por muchas (vs.1298), prueuo en aprueuo (vs. 1339), marcos en martos (vs.2295), priego en pliego (vs.2306), y tacto en tactos (vs.2356). El comentarista utilizó la edición de Granada (1505), porque en la edición de Sevilla (1499) se lee en el vs. 48 cobdiciando.
} 
que encontró en su fuente (De imagine mundi, cap. XXXV: "Haec et Trinacria a tribus montibus dicitur. In hac est mons Aethna cujus sulfurea exaestuant incendia”) con la descripción que Ovidio hace de Sicilia en sus Metamorfosis, V, 346 y ss.:

Vasta giganteis ingesta est insula membris Trinacris et magnis subiectum molibus urguet aetherias ausum sperare Typhoea sedes, nititur ille quidem pugnatque resurgere saepe, dextra sed Ausonio manus est subiecta Peloro, laeua, Pachyne, tibi, Lilybaeo crura premuntur, degrauat Aetna caput, sub qua resupinus harenas eiectat flammam-que fero uomit ore Typhoeus.

Mena reduce los cuatro montes a tres "para ajustarse a la etimología de 'Trinacria con sus tres altares". ${ }^{21}$ En sus ediciones del Laberinto Cummins y Pérez Priego prefieren la lectura Lelibeo (por lilibeo) del ms. PN7 a Edneo. Sin embargo, es evidente que las fuentes, De imagine mundi y Ovidio, apoyan la última lección: el monte Etna cubre la cabeza del gigante Tifeo, que vomita torrentes de arena y fuego. Además, la lectura lilibeo de PN7, que es lección única, es el resultado de una corrección de edneo, y con toda probabilidad es de la mano del comentarista A. Este erudito, continuando la glosa del copista A sobre Trinacria en el margen izquierdo, anota: "El vno Peloro es de la parte de Messina; Pachino de la parte de Siracusa (?); Lilibeo de la parte de Marsala. Virgilio en [el] fin del terçero libro"; y en el margen derecho escribe el mismo comentarista, parafraseando los versos ovidianos:

Tyfeo. Este Tyfeo fue gigante muy feroçe e superbo, el qual tentó tomar e vsurpare el reyno del çielo. Júpiter lo fulminó y lo condepnó a estar debasso de los montes de Sicilia. Y yaze supino: la cabeza tyene debaxo del monte de Ethna, dicho Mongibello, por donde ynsufla y vomita muchas vezes fuego. La man[o] dextra tiene debaxo del monte de Peloro, la siniestra debasso de Pachyno, las piernas debaxo de Lilibeo. E muchas vezes tenta leuantarse, haze gran fuerça y faze temblar la tierra. Mas por el peso de tan grandes montes que tiene de ençima no se puede leuantar. Ouidio en el $\mathrm{V}^{\circ}$ descriuiendo esta ystoria dize las siguientes palabras: [siguen los versos de Ovidio].

${ }^{21}$ María Rosa Lida de Malkiel, Juan de Mena, 36. 


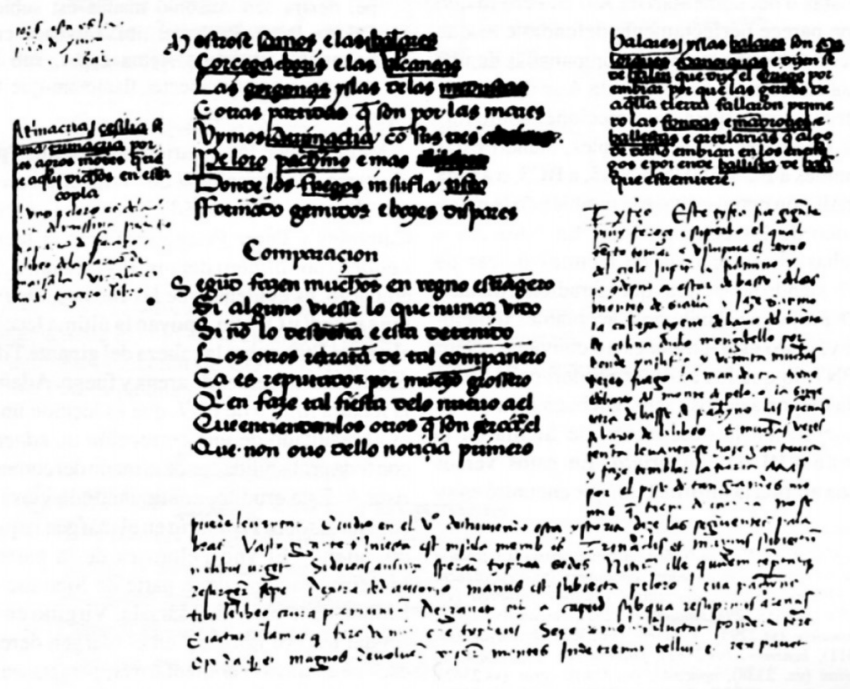

Con toda probabilidad prefirió el comentarista la lectura lilibeo por parecerle extraña la deformación edneo.

Las correcciones en PN7 son las siguientes:

4. si a (interlineado) si; fizo $\rightarrow$ se (inteirl.) fizo

12. lo $\rightarrow$ los ( $s$ sobrepuesta); quexoso $\rightarrow$ quexosos ( $s$ sobrep.)

13. aquel $[+\mathrm{BC} 3] \rightarrow$ que al (interl.) $[+$ glosa com. A]

15. de $[+\mathrm{BC} 3] \rightarrow$ de los (interl.) [com. A]

16. de $\rightarrow$ y de $[+$ glosa com. A]

19. por que $\rightarrow y$ por que

23. de gentes $\rightarrow$ de gente

35. no $[+\mathrm{NH} 5] \rightarrow$ nos

41. ya pues $\rightarrow$ e ya pues

48. cobdiciando; interl.: cobijando [com. B]

61. quin las $[+\mathrm{BC} 3] \rightarrow$ atlas $[+$ glosa com. A]

69. muestra $[+\mathrm{BC} 3, \mathrm{NH} 5] \rightarrow$ o muestra

81. las $[+\mathrm{BC} 3, \mathrm{NH} 5] \rightarrow \operatorname{los}[+$ glosa com. A]

82. $\operatorname{si} \rightarrow \sin$

85. norte; interl.: austro [com. A; + glosa com. A]

89. alli $[+\mathrm{BC} 3] \rightarrow$ assi

116. faro [+ los demás mss.] $\rightarrow$ paro [+ glosa com. A ]

125. muchos $[+\mathrm{NH} 5] \rightarrow$ mucho

136. abastante $[+\mathrm{BC} 3, \mathrm{NH} 5] \rightarrow$ ser bastante 
139. presura $[+$ los demás mss. $] \rightarrow$ spesura

140. tiene $[+\mathrm{NH} 5] \rightarrow$ çiñe

178. so (interl.) $\rightarrow$ soy

181. absençia $[+\mathrm{NH} 5] \rightarrow$ essençia

205. effecto $[+\mathrm{BC} 3, \mathrm{NH} 5] \rightarrow$ deffecto

212-213. [+ BC3, NH5]; el com. A cambia el orden mediante $b$-a

213. cabtela $[+\mathrm{BC} 3, \mathrm{NH} 5] \rightarrow$ captela

234. engora $\rightarrow$ engorra ( $r$ sobrep.)

237. estava $\rightarrow$ questava

274. persia $[+\mathrm{BC} 3, \mathrm{NH} 5] \rightarrow$ partia $[+$ glosacom. A $]$

293. librano $[+\mathrm{NH} 5] \rightarrow$ libiano (o: libano)

298. del $[+$ BC3, NH5] $\rightarrow$ al $[+$ glosa com. A $]$

326-327. [+ NH5]; com. A; b-a

326. e sauria $[+\mathrm{NH} 5] \rightarrow$ e isauria

334. ziffeos [+ los demás mss. (- MM1)] $\rightarrow$ riffeos [+ glosa com. A]

350. germonia $[+\mathrm{BC} 3, \mathrm{NH} 5] \rightarrow$ germania

355. catonia [+los demás mss. (-BM1 y CO1 $\rightarrow$ cahonia [+ glosa com. A]

382. busitania $[+\mathrm{BC} 3] \rightarrow$ lusitania $[+$ glosa com. A $]$

388. de lopia $\rightarrow$ dolopia [+ glosa com. A]

390. regoditas $[+\mathrm{NH} 5] \rightarrow$ tragoditas

398. getubia $[+\mathrm{NH} 5] \rightarrow$ getulia

399. fuellan [+ los demás mss. $\rightarrow$ pueblan

405. estogadas $[+\mathrm{NH} 5] \rightarrow$ escrofadas [+ glosa com. A: escrofadas]; nuue $\rightarrow$ nueue

406. e la $\rightarrow$ la

415. ycara $[+\mathrm{NH} 5] \rightarrow$ ycaro $[+$ glosa com. A $]$

418. bozis $[+$ los demás mss. $(-\mathrm{HH} 1)] \rightarrow$ ebozis $[+$ glosa com. A]

422. el edneo $[+$ los demás mss. (con variantes) $] \rightarrow$ lilibeo $[+$ glosa com. A]

431. entienden $[+\mathrm{NH} 5] \rightarrow$ entiendan

447. frente $\rightarrow$ fruente

462. futura $\rightarrow$ figura

475. uey $[+$ los demás mss. $(-$ SA5 $)] \rightarrow$ uee

482. faste - fasta

495. podra $[+$ los demás mss. (- ML2) $\rightarrow$ podria ( $i$ sobrep.)

518. el siglo $[+\mathrm{NH} 5] \rightarrow$ en (interl.) el siglo

550. casas $[+\mathrm{NH} 5] \rightarrow$ castas

563. tropos [+ los demás mss.] $\rightarrow$ an (interl.) tropos estr. 73-74. [+ NH5]; el com. A cambia el orden indicándolo con $b-a$

581. diestro $\rightarrow$ del ( interl.) diestro 
621. sy ouo $\rightarrow$ esyona

637. sinto $\rightarrow$ siento ( $e$ interl.)

684. fijo muerto $\rightarrow$ fijo ya (interl.) muerto

693. faleso $\rightarrow$ falsos ( $s$ sobrep.)

698. arceon [+ NH5: archeon; BC3, CO1, MH1, ML2, MM1, SM1, SV2: archero, BM1: rpa (sic), GB1: archeo, HH1, PN5: archeron, MN6b: arches, SA5: archion] $\rightarrow$ ericeon [+ glosa com. A]

709. aquelanthenor $\rightarrow$ aquel vil (interl.) anthenor

713. ensifile $[+\mathrm{NH} 5] \rightarrow$ erifile $[+$ glosa com. A]

719. ensifiles [ $+\mathrm{NH} 5] \rightarrow$ erisifiles

819. figo $\rightarrow$ figio $[+$ glosa com. A]

870. se $\rightarrow$ assi

228 871. frente $\rightarrow$ fruente

882. de $[+\mathrm{NH} 5] \rightarrow$ a las

893. deuia $[+\mathrm{NH} 5] \rightarrow$ deuiera

947. e senero $\rightarrow$ senero (se tachó la $e$ )

950. pluçio $\rightarrow$ interl.: planco [com. B]; apolidoro $\rightarrow$ interl.: apollodoro [com. B]

958. çatarizar $\rightarrow$ interl.: cytarizar [com. B]

968. delfyguieta $\rightarrow$ interl.: phrygineta [com. B]

1035. serita $[+\mathrm{NH} 5] \rightarrow$ erito

1105. metales $[\mathrm{cf} . \mathrm{NH} 5] \rightarrow$ meteles

1117. nucio [+ NH5] $\rightarrow$ mucio

1146. educos $[+\mathrm{NH} 5] \rightarrow$ etneos

1268. fallamo $\rightarrow$ fallamos ( $s$ sobrep.)

1275. que fondas $[+\mathrm{NH} 5] \rightarrow$ que no (interl.) fondas

1289. pisguerra $\rightarrow$ interl.: pisuerga [com. B]

1294. gibeltar $[+\mathrm{NH} 5] \rightarrow$ gibraltar

1297. sy $\rightarrow$ sy en (interl.)

1298. muchas $\rightarrow$ por muchas [com. B]

1308. dar $\rightarrow$ e dar

1335. gibaltar $\rightarrow$ gibraltar

1339. prueuo $\rightarrow$ aprueuo ( $a$ interl.) $[$ icom. B?]

1350. vieramos $[+$ los demás mss. $(-\mathrm{BM} 1)] \rightarrow$ lo (interl.) vieramos [com.B]

1354. lo $\rightarrow$ los

1521. pudo [+ BC3, NH5] $\rightarrow$ no (interl.) pudo estr. 196-190 (fol. 50r)-191192 (fol.50v)- 197 (fol. 51r); el com. A pone una $a$ en el margen izquierdo de la estr. 196, y una $b$ en el de la 197

1579. remedio $\rightarrow$ remiedo

1646. leuar $[+$ BC3, NH5] $\rightarrow$ lauar 
1699. salua $[+\mathrm{NH} 5] \rightarrow$ saluo

1725. toralato $(?) \rightarrow$ torquato $[+$ glosa com. A]

1844. introduziendo $[+\mathrm{NH} 5] \rightarrow$ introduzido; prio $[+\mathrm{NH} 5] \rightarrow$ pro

1884. dan $[+\mathrm{NH} 5] \rightarrow \mathrm{da}$

1907. que $[+\mathrm{NH} 5] \rightarrow \mathrm{y}$

1912. vengamos $\rightarrow$ non vengamos (non en el margen)

1921. del imçeo $\rightarrow$ de lynçeo [+ glosa com. A]

1922. yena [+ NH5] $\rightarrow$ hyena [+ glosa com. A]; nudo $\rightarrow$ nodo

1962. su dissono [+ los demás mss. $] \rightarrow \mathrm{y}$ dissono [+ glosa com. A]

1964. mienten $\rightarrow$ miecten

2000. tavida $\rightarrow$ tabida [+ glosa com. A]; luci da [+ NH5] $\rightarrow$ lurida

2034. partiçiparan $\rightarrow$ partir

2038. la ?ora $\rightarrow$ la yra

2043. subimo $\rightarrow$ sublimo

2060. su $[+\mathrm{NH} 5] \rightarrow \mathrm{la}$

2074. çesar $\rightarrow$ a (interl.) çesar

2085. como $\rightarrow$ e como

2087. caesçe $\rightarrow$ acaesçe ( $a$ sobrep.)

2098. fechos los ya [+ NH5] $\rightarrow$ los (antepues to) [icom. A?] fechos ya

2104. hauia $\rightarrow$ hauias (s sobrep.)

2134. vos $[+\mathrm{BC} 3, \mathrm{NH} 5] \rightarrow$ nos

2139. arizonte $[+\mathrm{NH} 5] \rightarrow$ orizonte

2192. de rayo dençima $\rightarrow$ mediante una $b$ y una $a$ el com. A indica el cambio

del orden de las palabras

2262. a liti; interl.: luque [com. B]

2279. que ${ }^{\wedge}$ de; en el margen: ^ fue [com. B]

2281. elui $[+\mathrm{NH} 5]$ elin $[i$ com. B? $]$

2282. de $[+\mathrm{NH} 5] \rightarrow \mathrm{e}$

2285. restante; en el margen: rescate [com. B]

2291. iran $\rightarrow$ ira (dos puntos bajo la $n$ )

2292. terçero $\rightarrow$ terçer (un punto bajo la $o$ )

2293. el caudete; interl.: alcaudete [com. B]

2295. marcos $[+\mathrm{NH} 5] \rightarrow \operatorname{martos}[i \operatorname{com} . \mathrm{B}$ ?]

2305. canfe; interl.: y cañete [com. B]

2306. priego $\rightarrow$ pliego [com. B]

2311. belcayce; interl.: bencayde [com. B]

2312. benarmegir; interl.: benemexi [com. B]

2332. veraçe; interl.: fatidica [com. B]

2338. como serian; interl.: quando serian [com B] 
2340. pagadọ; interl.: apacados [com. B]

2356. tacto $\rightarrow$ tactos [com. B]

2362. uostras; interl.: veras [com. B] estrofas CCXCVIII-CCC:

8. comentos; interl.: tormentos [com. B]

23. saber; interl.: plazer [com. B]

24. mando; interl.: mundo [com. B]

\section{BiBLIOGRAFÍA}

Aubrun, CH. V., "Inventaire des sources pour l'étude de la poésie castillane au Xve siècle”, Estudios dedicados a Menéndez Pidal, tomo IV, Madrid, 1953, 297-330.

Azáceta, José María (ed.), Juan de Mena. Antología de su obra poética, Barcelona: Clásicos Plaza y Janés, 1986.

Black, Robert, "Poetic Taste at the Aragonese Court in Naples", en John Geary (ed.), Florilegium Hispanicum, Medieval and Golden Age Studies Presented to Dorothy Clotelle Clarke, Madison: Hispanic Seminary of Medieval Studies, 1983, 167-174.

BofARUll y SANS, FRANCISCO DE, Heraldic Watermarks or 'La heráldica en la filigrana del papel', Hilversum, 1956.

BRIQUet, C.M., Les filigranes, ed. de Allan Stevenson, 4 vols., Amsterdam, 1968.

Cummins, John (ed.), El Laberinto de Fortuna, de Juan de Mena, Salamanca: Biblioteca Anaya, 1968; Madrid: Ediciones Cátedra, 1979.

DutTon, BriAn, et al., Catálogo-Índice de la poesía cancioneril del siglo XV, 2 vols., Madison: Hispanic Seminary of Medieval Studies, 1982.

El Laberinto de Fortuna, Madrid: Colección Austral, 1989.

Juan de Mena, Obras completas, Barcelona: Planeta/Autores Hispánicos, 1989.

Kerkhof, MaXim. P.A.M. (ed.), D. Iñigo López de Mendoza, Marqués de Santillana, La Comedieta de Ponza, Groningen, 1976.

Kerkhof, Maxim. P.A.M., "Hacia una nueva edición crítica del Laberinto de Fortuna de Juan de Mena”, Journal of Hispanic Philology, 7, 1983, 179-189.

Lida de MAlKiel, María Rosa, Juan de Mena, poeta del prerrenacimiento español, México, 1950.

Morel-Fatio, A., Catalogue des manuscrits espagnols et portugaises de la Bibliothèque Nationale, Paris, 1892.

OchoA, E. DE, Catálogo razonado de los MSS españoles existentes en la Biblioteca Real de París, Paris, 1844.

Pérez Priego, Miguel Angel (ed.), Juan de Mena, Laberinto de Fortuna, poemas menores, Madrid: Editora Nacional, 1976. 
Petrarca, Francesco, Le Rime, ed. de Giosuè Carducci y Severino Ferrari, Firenze: Sansoni, 1965.

Severin, Dorothy S., y Fiona Maguire, “The Spanish Songbook Project”, Journal of the Institute of Romance Studies, I, 1992, 49-57.

Street, Florence, "The Text of Mena's Laberinto in the Cancionero de Ixar and its Relationship to someother Fifteenth-Century MSS”, Bulletin of Hispanic Studies, 35, 1958, 63-71.

VAsvari Fainberg, Louise (ed.), El laberinto de Fortuna, de Juan de Mena, Madrid: Clásicos Alhambra, 1976.

Weiss, Julian, The Poet's Art. Literary Theory in Castile c. 1400-60, Oxford: Medium Aevum Monographs, New Series XIV, 1990.

Zingarelli, Nicola, Vocabulario della lingua italiana, Bologna, 1970 [10ª ed.]. 
